\title{
Diagnostic Accuracy of Magnetic Resonance Imaging for Nerve Injury in Obstetric Brachial Plexus Injury: Protocol for Systematic Review and Meta- analysis
}

Claire Madeline Hardie ( $\square$ clairemhardie@gmail.com )

Leeds Teaching Hospitals NHS Trust https://orcid.org/0000-0002-1271-6159

James Brooks

University of Leeds

Ryckie G Wade

University of Leeds

Irvin Teh

University of Leeds

Grainne Bourke

Leeds Teaching Hospitals NHS Trust

\section{Protocol}

Keywords: Brachial Plexus Neuropathies, Magnetic Resonance Imaging, Birth Injuries

Posted Date: October 21st, 2021

DOI: https://doi.org/10.21203/rs.3.rs-978809/v1

License: (c) (1) This work is licensed under a Creative Commons Attribution 4.0 International License.

Read Full License 


\section{Abstract}

\section{Background}

Obstetric brachial plexus injury (OBPI) is typically caused by excessive traction to the neck during delivery and can cause permanent dysfunction of the upper limb. Surgical exploration is typically performed at 3 months of age if insufficient signs of spontaneous functional recovery are demonstrated, and nerve reconstruction is performed at this point if indicated. Magnetic resonance imaging (MRI) offers a noninvasive alternative in detecting nerve injury, however the reported diagnostic accuracy in existing literature is conflicting. A systematic review is therefore required to clarify the accuracy of MRI and help define its role clinically. The primary objective is to determine the diagnostic accuracy of MRI for detecting root avulsion in obstetric brachial plexus injuries. The secondary objectives are to determine the diagnostic accuracy of MRI for detecting any nerve abnormality (including post-ganglionic injury) and to determine the diagnostic accuracy of MRI for detecting pseudomeningocele(s) as a surrogate marker of root avulsion.

\section{Methods}

Electronic databases will be searched systematically for studies reporting the accuracy of MRI (index test) compared to surgical exploration (reference standard) in detecting root avulsions, other nerve abnormalities and pseudomeningocele in children with OBPI. A revised QUADAS-2 tool will be used to assess methodological quality of included studies. If appropriate, summary sensitivities and specificities for target conditions (root avulsion, abnormal nerve and pseudomeningocele) will be obtained via metaanalyses using a bivariate model.

\section{Discussion}

This study will aim to clarify the current diagnostic accuracy of MRI for detecting nerve injury and define its clinical role.

Systematic review registration

PROSPERO registration CRD42021267629.

\section{Background}

\section{Obstetric brachial plexus injury}

Obstetric brachial plexus injury (OBPI) is defined as flaccid paralysis of the upper limb at birth (1) and is typically caused by excessive traction applied to the neck during delivery. OBPI affects 0.4 to 2 children per 1000 births (2). Risk factors include fetal macrosomia, shoulder dystocia, humeral fracture and vacuum or forceps evacuation $(3,4)$. Fracture of the clavicle during a dystocic delivery is often considered to be protective as the space between the clavicle and first rib is increased leading to reduced 
force on the brachial plexus as it leaves the thorax (5). However, some studies argue that a clavicle fracture is indicative of birth trauma and therefore the risk of OBPI is increased (6). All roots from C5-T1 can be damaged however C5 and C6 involvement (Erb's palsy) is most frequent $(7,8)$.

Spontaneous recovery is common within the first 3 months of life, however this recovery is incomplete in $10-30 \%$ of cases (9). Muscle changes in infants with permanent brachial plexus injuries can result in shoulder and elbow contractures (tightening of the muscles and tendons) which can lead to long term pain and loss of function (10). Joint deformity, weakness and limited movement at the wrist and hand may also occur with permanent OPBI $(11,12)$. An increased risk of psychological effects has been reported for affected infants and their mothers (13) as well as disruption to overall family functioning (14). In terms of hospital finances, the cost of babies born with OPBI is roughly twice as high (15). The lifetime costs are also significant when accounting for the cost of treatment, disability costs and loss of income.

The strongest prognostic factor that determines outcome is the extent of the primary plexus injury (16). Neuropraxia (stretching of the nerves) confers the best prognosis, nerve rupture more severe, and root avulsion (nerve roots pulled away from the spinal cord) the worst (17). The Sunderland classification can be used to define the severity of the injury, ranging from first degree (loss of axon conduction) to fifth degree (loss of continuity of the entire nerve trunk) (18). Hardly any functional recovery is observed with fourth and fifth degree injury (19).

Infants and children with limited to no functional recovery can benefit from surgical intervention with nerve grafting or transfers. Nerve transfer is used in cases of pre-ganglionic injury (root avulsion) which involves redirecting functioning nerves in the neck to supply the arm (20). Post-ganglionic injuries are managed with nerve grafts which involve harvesting less important nerves (e.g. the sural nerve) and using them to restore continuity in the ruptured nerve. In both cases early reconstructive nerve surgery is associated with better functional recovery (21).

Whilst scoring systems such as the Active Movement Scale (22) can be used to aid in assessment, it is still difficult to clinically determine the extent of the injury.

\section{Diagnostic modalities of interest}

Imaging can be used to support decision making in obstetric brachial plexus surgery. Clinical practice has moved away from computed tomography myelography in the evaluation of OBPI and towards magnetic resonance imaging (MRI) (23) which has clear advantages when it comes to imaging children such as the absence of ionising radiation and intrathecal contrast agent. Enhanced soft tissue visualisation and multiplanar reconstructions also make MRI a suitable modality. MRI is currently thought to be the best non-invasive test for imaging the brachial plexus and detecting nerve root avulsion (24), but has been demonstrated to have variable sensitivity (63\%-88\%) and specificity (89\%-100\%) for detecting preganglionic injury and sensitivity $60-75 \%$ and specificity $89-100 \%$ for post-ganglionic injury $(23,25-28)$ when compared to surgical exploration. Pseudomeningoceles from leakage of cerebrospinal fluid can 
additionally be used as a surrogate marker of nerve root avulsion on MRI scans but again the reported accuracy of this is variable (29).

Due to the only moderate sensitivity of MRI to detect pre and post-ganglionic injuries this cannot be relied on to fully inform clinical decisions. It is critical to differentiate between pre-ganglionic and postganglionic injuries due to the differing prognosis and surgical approaches for these injuries. There is a pressing need to define the characteristics of the nerve injury that has potential to recover with reconstructive surgery. Post-ganglionic injuries are associated with better outcomes because the anterior horn cells are unaffected, meaning that motor function can be recovered if nerve continuity is restored $(30,31)$. Pre-ganglionic injuries confer the worst prognosis and require nerve transfer rather than grafting because the native motor neurons recede (32). Currently the only way to be certain of the extent of injury is to perform a surgical exploration at which point nerve reconstruction can be performed if necessary. Surgical exploration is typically indicated if there is no or limited improvement in biceps or deltoid function at 3 months of age (33).

1. Surgical exploration of the brachial plexus

Involves an incision in the supraclavicular fossa which enables direct visualisation of the roots C5T1 (under general anaesthetic).

2. MRI

Produces detailed images of the brachial plexus (also performed under general anaesthetic due to the young age of patients)

\section{Clinical pathway}

Diagnosis of OPBI is usually made at birth or soon after. Once diagnosed, a referral to physiotherapy for stretching and exercises is advised. The physiotherapist will manage the child for several weeks, looking for signs of functional recovery. If spontaneous recovery occurs then the child will be discharged. However, a referral to specialist services is required if the limb remains flaccid before the infant is 12 weeks of age. A specialist assessment involves performing a detailed history and examination, noting factors such as birth weight, shoulder dystocia, assisted delivery, Apgar scores (heart rate, respiratory effort, muscle tone, reflex and colour), presence of Horner's sign, hemi-diaphragm paralysis, presence of fractures and post-natal recovery of the affected arm. Scoring systems such as the Toronto score (34) are also used to classify functional status. Surgical exploration of the brachial plexus is indicated in children who fall into moderate or severe injury categories, and MRI can be performed to provide information prior to this.

\section{Prior tests}

Some babies will have had X-Rays performed of the upper limbs to examine for fractures and some may not have. Other tests for example ultrasound of the shoulders or blood tests may have also been 
performed.

\section{Importance of this review}

A highly sensitive and specific imaging modality that could clearly define the extent of the brachial plexus injury would enable earlier surgical intervention in those that require it, as well as limiting numbers of unnecessary surgical explorations. The reliability of MRI in detecting root avulsion and other nerve abnormalities is uncertain, therefore assessing the current accuracy of MRI for obstetric brachial plexus injuries will help define its role clinically and suggest areas for development. In existing literature, the reported diagnostic accuracy of MRI for obstetric brachial plexus injuries is variable and is often based on clinical follow-up (23) or electrophysiology (35). This review aims to clarify the overall diagnostic accuracy by comparing MRI to surgical exploration as a reference standard.

\section{Objectives}

1. To determine the diagnostic accuracy of MRI for detecting root avulsion in obstetric brachial plexus injuries

2. To determine the diagnostic accuracy of MRI for detecting abnormal nerves in obstetric brachial plexus injuries

3. To determine the diagnostic accuracy of MRI for detecting pseudomeningocele(s) as a surrogate marker of root avulsion in obstetric brachial plexus injuries

\section{Methods/ Design}

The Cochrane Handbook for Reviews of Diagnostic Test accuracy was used as guidance to write the methods (36) and the preferred reporting items for systematic reviews and meta-analyses (PRISMA) (37) guidelines were followed.

\section{Types of studies}

Studies involving infants with obstetric brachial plexus injuries that report the findings of preoperative MRI in comparison to surgical exploration of the brachial plexus roots will be included. Case reports will be excluded.

\section{Participants}

This review will include studies involving children under five years old with obstetric brachial plexus injuries. All injuries to the brachial plexus that occur during delivery will be included, irrelevant of the aetiology (e.g. shoulder dystocia, forceps delivery etc.) Bilateral injuries will also be included.

\section{Index Test}


The role of MRI will be to detect root avulsions, other nerve abnormalities and pseudomeningoceles. In OBPI MRI is typically performed at 3 months in infants with persistent upper limb functional limitations. The MRI acquisition will vary in terms of the physical scanner used (manufacturer and model), field strength, pulse sequences, coil arrangement, gradients, postprocessing techniques and other factors - all of which will impact upon image quality and hence diagnostic accuracy. Variations will also arise due to the subjective nature of image interpretation. A radiologist will review the images and either confirm or exclude the presence of avulsion, other nerve abnormality or pseudomeningocele. Positive findings for avulsion are detected by a lack of continuity or absence of the nerve root between the spinal cord and exit foramen (29). Other nerve abnormalities than may be detected include nerve scarring, neuroma or rupture, and these can also be referred to as post-ganglionic nerve injuries $(25,27)$. An abnormal contour of the dura and collection of dorsal extraspinous fluid is indicative of pseuomeningocele (38) and is considered a surrogate marker of root avulsion given that rupture of the dura mater suggests that the nerve root is also ruptured, although this has been disputed in some literature $(39,40)$. The presence of one suspected avulsion is of equal importance to that of any number of avulsions, given that any avulsion would warrant nerve transfer surgery. Due to the lower energy stretching forces that typically cause OBPI other types of severe nerve injury apart from avulsion may also be present and can also require surgery e.g. nerve grafting. Avulsions, other nerve injury and pseudomeningoceles can occur at any spinal level from C4 to T2 and may, in rare cases, occur bilaterally. The ability of MRI to identify patients with no root avulsion is vital as surgical exploration could potentially be avoided.

\section{Target condition}

Avulsion of the roots of the brachial plexus is the target condition. The ability of MRI to differentiate between any number of root avulsions and no avulsions will be examined. The secondary target conditions are an abnormal nerve and pseudomeningocele.

\section{Reference standard}

Surgical exploration of the brachial plexus is the reference standard for detecting root avulsion and other nerve abnormalities. The operation is performed under general anaesthesia and involves an incision in the supraclavicular fossa which extends towards the deltopectoral groove (29). The operation allows direct inspection of the spinal nerve roots C4-T1. Additional intraoperative tests such as somatosensory evoked potentials (SEPs) and bipolar motor nerve stimulation are included as part of the reference standard. SEPs involve measuring cortical activity induced by applying pulses of varying frequency to the nerve roots. Avulsed nerves will not transmit signals to the brain meaning no activity is detected on an encephalogram. Bipolar nerve stimulation involves applying a current across the nerve which would normally cause the corresponding muscle to contract, however in the case of avulsion, no muscle contraction is observed. These intraoperative tests aid surgeons in the diagnosis of root avulsion.

\section{Search strategy}

Electronic searches 
EMBASE, PubMed and CENTRAL electronic databases will be searched from inception to present date with no restrictions. The medRxiv and bioRxiv preprint archives will also be searched using medrixr (41). GScraper will be used to further increase coverage by pulling hits from Google Scholar (42). The planned EMBASE and PubMed search strategy was formulated with a search strategist and is presented in appendix 1.

\section{References from published studies}

Citations will be imported and de-duplicated using EndNote. References will then will be screened using CitationChaser (43).

\section{Study selection}

JB will apply the eligibility criteria to screen titles and abstracts for relevance. Two authors (JB and $\mathrm{CH}$ ) will independently screen identified citations using Rayyan REF. Disagreements will be resolved by discussion with a third author (GB). Full text of eligible studies will then be screened and subsequently labelled as included or excluded. Reasons for exclusion will be noted.

\section{Data Extraction}

For included studies the following data will be extracted using an excel spreadsheet. This will be done independently in duplicate by JB and $\mathrm{CH}$ : study identifier; number of participants; sex; participants age at diagnosis, MRI scan and surgery; country of origin; time from birth to imaging and surgery; presence of clavicular/humeral fracture; type of MRI scanner used (including brand, model and field strength); pulse sequence; number of true positives, false positives, true negatives and false negatives relating to the detection of root avulsion, abnormal nerve or pseudomeningocele using MRI. The priority outcome is the detection of root avulsion at the brachial plexus as this is the is most important clinically. Detection of abnormal nerves and pseudomeningocele are secondary outcomes. We will contact authors of studies by email if data is missing or unclear.

\section{Methodological quality assessment}

An adapted version of the Quality Assessment of Diagnostic Accuracy Studies (QUADAS-2) (44) tool (Appendix 2) will be used to assess the risk of bias and strength of evidence of the eligible studies respectively at the study level. Two authors (JB and $\mathrm{CH}$ ) will independently complete the assessment. Disagreements will be resolved by discussion with a third author (GB).

\section{Data synthesis}

Analysis will be performed separately for each target condition (root avulsion, abnormal nerve and pseudomeningocele) with the patient and then the nerves being the unit of analysis. Forest plots and receiver operating characteristic plots will be used to display estimates of sensitivity and specificity of the included studies as part of the preliminary analysis. These plots will be generated using MetaDTA (45). 
Summary sensitivities and specificities will be obtained using a bivariate model for meta-analyses, providing data is sufficient (46). Meta-analyses will be performed using Stata version 15 (47).

\section{Investigations of heterogeneity}

Heterogeneity in the diagnostic accuracy of MRI will be examined using meta-regression or subgroup analyses if data permits. Variations in field strength is likely to be a source of heterogeneity (29) and will be investigated. Babies with OPBI who have not undergone surgical exploration (e.g. they are too unwell) may result in underestimation of the number false negatives which in turn could upwardly bias the sensitivity of MRI. Furthermore, the diagnostic accuracy of MRI could be biased by retrospective studies which have recruited an unrepresentable sample of patients.

\section{Sensitivity analysis}

The impact of bias will be evaluated via sensitivity analyses. Studies with a high or unclear risk of bias as identified by the tailored QUADAS-2 tool will be excluded.

\section{Assessment of reporting bias}

Reporting bias will not be assessed given the lack of sensitive statistical methods (36). Data will be made available on the Open Science Framework.

\section{Discussion}

OBPI can lead to significant morbidity both in terms of the physical implications (e.g. loss of function) and psychological impact $(13,48)$. MRI offers a non-invasive method of visualising the brachial plexus which could potentially reduce the number of infants undergoing surgery and facilitate earlier treatment in those that require it. However, the reported sensitivity and specificity of MRI in detecting root avulsion and other nerve injuries is variable. A systematic review is required to determine the diagnostic accuracy of MRI in detecting nerve injury in infants with $\mathrm{OBPI}$ and define its role clinically. We anticipate variability in scanning techniques, scan reporting as well as surgical techniques and reporting of findings. There is likely to also be a mix of prospective and retrospective studies. We also anticipate possible presence of bias in included studies, for example non-consecutive recruiting or reporting only of positive findings MRI findings. The quality and variability of the included studies will be carefully evaluated using the methods described and results interpreted appropriately.

\section{List Of Abbreviations}

OBPI Obstetric Brachial Plexu Injury

MRI Magnetic Resonance Imaging

QUADAS-2 Quality Assessment of Diagnostic Accuracy Studies 


\section{Declarations}

Ethics approval and consent to particiate

Not applicable

Consent for publication

Not applicable

Availability of data and materials

Data sharing is not applicable to this article as no datasets were generated or analysised. Materials (Search strategy and QUADAS-2 tool) are available in Appendix 1 and Appendix 2.

\section{Funding}

Ryckie Wade is supported by the National Institute for Health Research (NIHR) in Leeds (DRF-2018-11ST1-159). The views expressed are those of the author(s) and not necessarily those of the United Kingdom's National Health Service, NIHR or Department of Health and Social Care.

Competing interests

The authors declare they have no competing interests.

Authors Contributions

$\mathrm{CH}, \mathrm{JB}$ and $\mathrm{GB}$ conceived the study. $\mathrm{CH}$ and JB drafted and revised the protocol, designed data collection tools and the statistical analysis plan with input from GB, RW and IT. $\mathrm{CH}$ is the guarantor of the review.

Acknowledgements

Not applicable.

\section{References}

1. Andersen J, Watt J, Olson J, Van Aerde J. Perinatal brachial plexus palsy. Paediatrics \& child health. 2006;11(2):93-100.

2. Lombard A, Bachy M, Fitoussi F. C5-8 neonatal brachial plexus palsy. Operative findings, reconstructive strategy and outcome. 2020;45(8):798-804.

3. Sibiński M, Synder M. Obstetric brachial plexus palsy-risk factors and predictors. Ortop Traumatol Rehabil. 2007;9(6):569-76.

4. Foad SL, Mehlman CT, Ying J. The epidemiology of neonatal brachial plexus palsy in the United States. J Bone Joint Surg Am. 2008;90(6):1258-64. 
5. Gandhi RA, DeFrancesco CJ, Shah AS. The Association of Clavicle Fracture With Brachial Plexus Birth Palsy. The Journal of Hand Surgery. 2019;44(6):467-72.

6. Coroneos CJ, Voineskos SH, Coroneos MK, Alolabi N, Goekjian SR, Willoughby LI, et al. Obstetrical brachial plexus injury: burden in a publicly funded, universal healthcare system. Journal of Neurosurgery: Pediatrics PED. 2016;17(2):222-9.

7. Erb WH. Ueber eine eigenthümliche Localisation von Lähmungen im Plexus brachialis: Carl Winter's Universitätbuchhandlung; 1874.

8. GILBERT A, WHITAKER I. Obstetrical Brachial Plexus Lesions. Journal of Hand Surgery. 1991;16(5):489-91.

9. Terzis JK, Kokkalis ZT. Pediatric brachial plexus reconstruction. Plast Reconstr Surg. 2009;124(6 Suppl):e370-e85.

10. Gharbaoui IS, Gogola GR, Aaron DH, Kozin SH. Perspectives on glenohumeral joint contractures and shoulder dysfunction in children with perinatal brachial plexus palsy. J Hand Ther. 2015;28(2):17683; quiz 84.

11. Strömbeck C, Krumlinde-Sundholm L, Forssberg H. Functional outcome at 5 years in children with obstetrical brachial plexus palsy with and without microsurgical reconstruction. Dev Med Child Neurol. 2000;42(3):148-57.

12. Waters PM. Update on management of pediatric brachial plexus palsy. J Pediatr Orthop B. 2005;14(4):233-44.

13. Alyanak B, Kılınçaslan A, Kutlu L, Bozkurt H, Aydın A. Psychological Adjustment, Maternal Distress, and Family Functioning in Children With Obstetrical Brachial Plexus Palsy. The Journal of Hand Surgery. 2013;38(1):137-42.

14. Fırat T, Oskay D, Akel BS, Öksüz Ç. Impact of obstetrical brachial plexus injury on parents. Pediatrics International. 2012;54(6):881-4.

15. Wali AR, Santiago-Dieppa DR, Brown JM, Mandeville R. Nerve transfer versus muscle transfer to restore elbow flexion after pan-brachial plexus injury: a cost-effectiveness analysis. Neurosurgical Focus FOC. 2017;43(1):E4.

16. Kirjavainen M, Remes V, Peltonen J, Kinnunen P, Pöyhiä T, Telaranta $T$, et al. Long-term results of surgery for brachial plexus birth palsy. J Bone Joint Surg Am. 2007;89(1):18-26.

17. Bahm J, Ocampo-Pavez C, Disselhorst-Klug C, Sellhaus B, Weis J. Obstetric brachial plexus palsy: treatment strategy, long-term results, and prognosis. Deutsches Arzteblatt international. 2009;106(6):83-90.

18. Sunderland S. A classification of peripheral nerve injuries producing loss of function. Brain. 1951;74(4):491-516.

19. Hoeksma AF, ter Steeg AM, Nelissen RG, van Ouwerkerk WJ, Lankhorst GJ, de Jong BA. Neurological recovery in obstetric brachial plexus injuries: an historical cohort study. Dev Med Child Neurol. 2004;46(2):76-83. 
20. Malessy MJ, de Ruiter GC, de Boer KS, Thomeer RT. Evaluation of suprascapular nerve neurotization after nerve graft or transfer in the treatment of brachial plexus traction lesions. J Neurosurg. 2004;101(3):377-89.

21. Terzis JK, Papakonstantinou K. Surgical Treatment of Obstetrical Brachial Plexus Paralysis: The Norfolk Experience. Seminars in Plastic Surgery. 2004;18(4):359-75.

22. Curtis C, Stephens D, Clarke HM, Andrews D. The active movement scale: an evaluative tool for infants with obstetrical brachial plexus palsy. J Hand Surg Am. 2002;27(3):470-8.

23. Grahn P, Pöyhiä T, Sommarhem A, Nietosvaara Y. Clinical significance of cervical MRI in brachial plexus birth injury. Acta Orthop. 2019;90(2):111-8.

24. Vargas MI, Viallon M, Nguyen D, Beaulieu JY, Delavelle J, Becker M. New approaches in imaging of the brachial plexus. Eur J Radiol. 2010;74(2):403-10.

25. Gad DM, Hussein MT, Omar NNM, Kotb MM, Abdel-Tawab M, Yousef HAZ. Role of MRI in the diagnosis of adult traumatic and obstetric brachial plexus injury compared to intraoperative findings. Egyptian Journal of Radiology and Nuclear Medicine. 2020;51(1):195.

26. Medina LS, Yaylali I, Zurakowski D, Ruiz J, Altman NR, Grossman JAl. Diagnostic performance of MRI and MR myelography in infants with a brachial plexus birth injury. Pediatric Radiology. 2006;36(12):1295-9.

27. Gunes A, Bulut E, Uzumcugil A, Oguz KK. Brachial Plexus Ultrasound and MRI in Children with Brachial Plexus Birth Injury. 2018;39(9):1745-50.

28. Somashekar D, Yang LJ, Ibrahim M, Parmar HA. High-resolution MRI evaluation of neonatal brachial plexus palsy: A promising alternative to traditional CT myelography. AJNR Am J Neuroradiol. 2014;35(6):1209-13.

29. Wade RG, Takwoingi Y, Wormald JCR, Ridgway JP, Tanner S, Rankine JJ, et al. Magnetic resonance imaging for detecting root avulsions in traumatic adult brachial plexus injuries: protocol for a systematic review of diagnostic accuracy. Systematic Reviews. 2018;7(1):76.

30. Dubuisson AS, Kline DG. Brachial plexus injury: a survey of 100 consecutive cases from a single service. Neurosurgery. 2002;51(3):673-82; discussion 82-3.

31. Siqueira MG, Martins RS. Surgical treatment of adult traumatic brachial plexus injuries: an overview. Arq Neuropsiquiatr. 2011;69(3):528-35.

32. Dolan RT, Butler JS, Murphy SM, Hynes D, Cronin KJ. Health-related quality of life and functional outcomes following nerve transfers for traumatic upper brachial plexus injuries. Journal of Hand Surgery (European Volume). 2011;37(7):642-51.

33. Smith NC, Rowan P, Benson LJ, Ezaki M, Carter PR. Neonatal brachial plexus palsy. Outcome of absent biceps function at three months of age. J Bone Joint Surg Am. 2004;86(10):2163-70.

34. Michelow BJ, Clarke HM, Curtis CG, Zuker RM, Seifu Y, Andrews DF. The natural history of obstetrical brachial plexus palsy. Plast Reconstr Surg. 1994;93(4):675-80; discussion 81. 
35. van Ouwerkerk WJ, Strijers RL, Barkhof F, Umans U, Vandertop WP. Detection of root avulsion in the dominant $\mathrm{C} 7$ obstetric brachial plexus lesion: experience with three-dimensional constructive interference in steady-state magnetic resonance imaging and electrophysiology. Neurosurgery. 2005;57(5):930-40; discussion -40.

36. Collaboration TC. The Cochrane Handbook for Diagnostic Test Accuracy Reviews 2016 [Available from: http://methods.cochrane.org/sdt/handbook-dta-reviews.

37. Moher D, Liberati A, Tetzlaff J, Altman DG, Group P. Preferred reporting items for systematic reviews and meta-analyses: the PRISMA statement. PLoS medicine. 2009;6(7):e1000097.

38. Sureka J, Cherian RA, Alexander M, Thomas BP. MRI of brachial plexopathies. Clin Radiol. 2009;64(2):208-18.

39. van Es HW, Bollen TL, van Heesewijk HPM. MRI of the brachial plexus: A pictorial review. European Journal of Radiology. 2010;74(2):391-402.

40. Laohaprasitiporn P, Wongtrakul S, Vathana T, Limthongthang R, Songcharoen P. Is Pseudomeningocele an Absolute Sign of Root Avulsion Brachial Plexus Injury? J Hand Surg Asian Pac Vol. 2018;23(3):360-3.

41. Schmidt LAMaL. medrxivr: Accessing and searching medRxiv and bioRxiv preprint data in R. Journal of Open Source Software. 2020;5(54):2651.

42. Haddaway N. GScraper 2020 [Available from: https://github.com/nealhaddaway/GSscraper.

43. Haddaway NR, Grainger MJ, Gray CT. citationchaser: an R package for forward and backward citations chasing in academic searching. 0.0.3 ed2021.

44. Whiting P, Rutjes AW, Reitsma JB, Bossuyt PM, Kleijnen J. The development of QUADAS: a tool for the quality assessment of studies of diagnostic accuracy included in systematic reviews. BMC Med Res Methodol. 2003;3:25.

45. Freeman SC, Kerby CR, Patel A, Cooper NJ, Quinn T, Sutton AJ. Development of an interactive webbased tool to conduct and interrogate meta-analysis of diagnostic test accuracy studies: MetaDTA. BMC medical research methodology. 2019;19(1):1-11.

46. Reitsma JB, Glas AS, Rutjes AW, Scholten RJ, Bossuyt PM, Zwinderman AH. Bivariate analysis of sensitivity and specificity produces informative summary measures in diagnostic reviews. J Clin Epidemiol. 2005;58(10):982-90.

47. StataCorp. Stata 152017 [Available from: https://www.stata.com/stata15/.

48. Gharbaoui I, Gogola GR, Aaron DH, Kozin SH. Perspectives on glenohumeral joint contractures and shoulder dysfunction in children with perinatal brachial plexus palsy. 2014;28(2):176-84.

\section{Supplementary Files}

This is a list of supplementary files associated with this preprint. Click to download.

- Appendices.docx 
- PRISMAPchecklist.docx

Page 13/13 